\title{
Inadvertent complication of the Senning procedure
}

\author{
S. Moustafa ${ }^{1}$ N. Merchant $^{2}$ M. S. Connelly ${ }^{3}$ D. J. Patton ${ }^{4}$
}

Published online: 23 May 2016

(c) The Author(s) 2016. This article is available at SpringerLink with Open Access

A 34-year-old man with complete transposition of the great arteries (D-TGA) presented for the first time to our clinic. He previously underwent balloon atrial septostomy (Rashkind procedure) early after birth followed by a Senning procedure at the age of 2 months together with ligation of a patent ductus arteriosus. He has been asymptomatic since his surgery.

Transthoracic echocardiogram was technically challenging yet revealed a mildly dilated, hypertrophied systemic right ventricle with mild systolic dysfunction, normal subpulmonary left ventricular size and systolic function with no baffle leak or stenosis. No significant valvulopathy was noted (Movie). Cardiovascular magnetic resonance (CMR) and cardiac computed tomography (CCT) showed a moderately hypertrophied systemic right ventricle with normal systolic function (right ventricular ejection fraction $48 \%$ ). The sub-pulmonic left ventricle was dilated with normal systolic function. Systemic venous baffles were patent. The pulmonary venous portion of the baffle was patent.

Electronic supplementary material The online version of this article (doi:10.1007/s12471-016-0840-8) contains supplementary material, which is available to authorized users.

D. J. Patton

David.Patton@albertahealthservices.ca

1 Division of Cardiovascular Diseases, Mayo Clinic Arizona, Scottsdale, AZ, USA

2 Department of Radiology, University of Calgary, Calgary, AB, Canada

3 Adult Congenital Heart Disease Clinic, University of Calgary, Calgary, AB, Canada

4 Section of Pediatric Cardiology, Alberta Children's Hospital, University of Calgary, Calgary, AB, Canada
The main pulmonary artery and both branches were dilated (Fig. 1).

CMR angiography and CCT uncovered a tortuous varix of the left lower pulmonary vein (PV) draining superiorly to a larger common left-side PV which in turn connected to the superior aspect of the left atrium to the left of the Senning baffle (residual left-to-right shunt). Qp:Qs ratio was estimated at 1.9 by flow data and 2.1 by stroke volume analysis. The right PVs were dilated (Fig. 1).

Due to the presence of significant residual left-toright shunt with a dilated sub-pulmonic ventricle, cardiac catheterisation and potential corrective surgery were recommended. However, the patient declined the procedure.

To the best of our knowledge, this is the first case in the literature exploring an unusual anomalous PV connection in a patient with D-TGA following atrial switch procedure. We speculate that the patient had this anomalous connection since birth but may have been overlooked during surgical correction [1-3].

Conflict of interest S. Moustafa, N. Merchant, M.S. Connelly and D.J. Patton state that there are no conflicts of interest.

Open Access This article is distributed under the terms of the Creative Commons Attribution 4.0 International License (http:// creativecommons.org/licenses/by/4.0/), which permits unrestricted use, distribution, and reproduction in any medium, provided you give appropriate credit to the original author(s) and the source, provide a link to the Creative Commons license, and indicate if changes were made. 

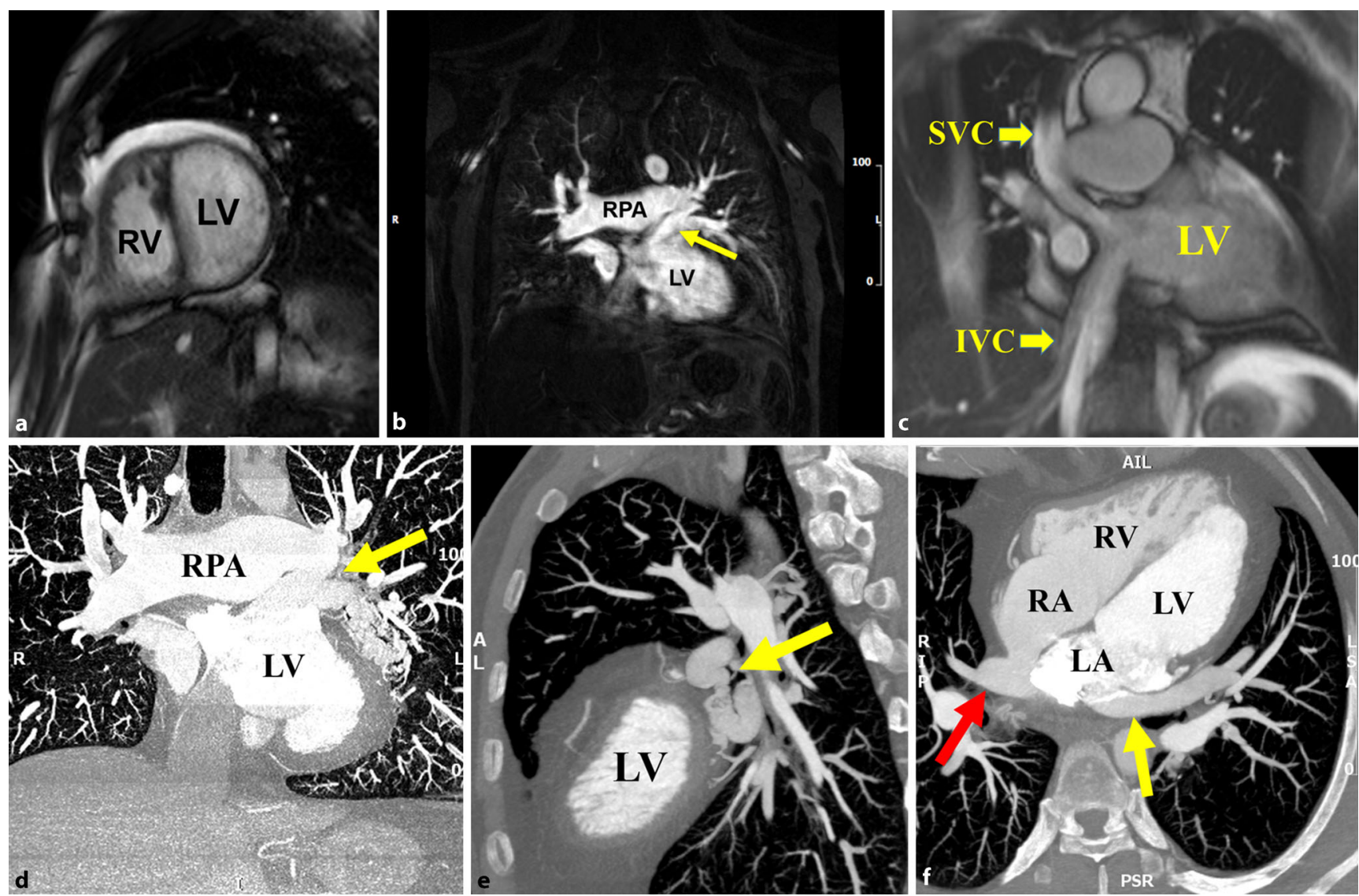

Fig. 1 a CMR short-axis steady-state free precession (SSFP) coronal image showing hypertrabeculated systemic RV with dilated LV. b CMR angiography revealing the left lower PV draining superiorly to a larger common left-side PV which in turn connects to the superior aspect of the left atrium (arrow). Right pulmonary artery (RPA) is dilated. c CMR SSFP coronal image revealing patent superior (SVC) and inferior vena cava (IVC) limbs of the Senning baffle. d CCT maximal intensity projection coronal image showing a tortuous varix of the left lower PV draining superiorly to a larger common left-side PV which in turn connects to the superior aspect of the left atrium. RPA is dilated. e CCT sagittal view revealing the tortuous varix (arrow). f CCT axial view showing the site of anastomosis between the left atrium and anomalous PV connection (yellow arrow). Patent pulmonary venous baffle was noted (red arrow)

\section{References}

1. Talwar S, Rajashekar P, Reddy VA, et al. Transposition of great arteries and partial anomalous pulmonary venous drainage. Asian Cardiovasc Thorac Ann. 2013;21:713-716.

2. Seliem MA, Bouholaigah IH, Palileo MR. Complete transposition of the great arteries and total anomalous pulmonary venous connection with a small atrial septal defect: a rare combination resulting in balanced pulmonary systemic circulations. Ann Saudi Med. 2004;24:133-135.

3. Ueda Y, Miki S, Okita Y, et al. Transposition of the great arteries associated with total anomalous pulmonary venous return. Ann Thorac Surg. 1994;57:470-472. 